\title{
Uterine Tonus Assessment by Midwives versus Patient self-assessment in the active management of the third stage of labor (UTAMP): study protocol for a randomized controlled trial
}

\author{
Joyce L. Browne ${ }^{1 *}$, Nelson K. R. Damale ${ }^{2,3}$, Tessa M. Raams ${ }^{1}$, Eva L. Van der Linden ${ }^{1}$, Ernest T. Maya ${ }^{2,4}$, \\ Roseline Doe ${ }^{3,5}$, Marcus J. Rijken ${ }^{1,6}$, Richard Adanu ${ }^{4}$, Diederick E. Grobbee ${ }^{1}$, Arie Franx ${ }^{6}$ and \\ Kerstin Klipstein-Grobusch ${ }^{1,7}$
}

\begin{abstract}
Background: Postpartum hemorrhage (PPH) is the leading cause of maternal mortality worldwide and accounts for one third of maternal deaths in low-income and middle-income countries. PPH can be prevented by active management of the third stage of labor (AMTSL), a series of steps recommended by the World Health Organization to be performed by skilled birth attendants (SBAs). Task shifting in the AMTSL step of uterotonic drugs administration to community health workers, traditional birth attendants and self-administration has been investigated as a strategy to increase access to quality obstetric care considering persistent SBA and facility-based delivery shortages. The aim of this study is to assess task shifting in the final step of AMTSL and compare uterine tonus assessment by a SBA to selfassessment.

Methods and Design: The study is an individual-level two-arm non-inferiority randomized controlled trial (RCT). A total of 800 women will be recruited in Korle Bu Teaching Hospital in Accra, Ghana. Adult women in labor at term with an expected vaginal delivery who received antenatal instructions for self-assessment of uterine tonus will be eligible for inclusion. Women with an increased risk for PPH will be excluded. Women will be randomized to uterine tone assessment by a skilled birth attendant (midwife) or uterine tone self-assessment (with the safety back-up of a midwife present in case of PPH or uterine atony). Postpartum blood loss will be measured through weighing of disposable mats. The main study endpoints are PPH ( $\geq 500 \mathrm{ml}$ blood loss), severe PPH ( $\geq 1000 \mathrm{ml}$ blood loss), mean blood loss, and routine maternal and neonatal outcomes. Participants and caregivers will not be blinded given the nature of the intervention.

Discussion: A reduction of PPH-related maternal mortality requires full implementation of AMTSL. Task shifting of uterine tone assessment may contribute to increased AMTSL implementation in (clinical) settings where SBAs capacity is constrained.

\footnotetext{
* Correspondence: J.L.Browne@umcutrecht.n

1 Julius Global Health, Julius Center for Health Sciences and Primary Care, University Medical Center Utrecht, Heidelberglaan 100, 3584CX, Utrecht, The Netherlands

Full list of author information is available at the end of the article
} 
(Continued from previous page)

Trial registration: Clinicaltrials.gov: NCT02223806, registration August 2014. PACTR: PACTR201402000736158, registration July 2014. University of Ghana, Medical School Ethical and Protocol Review Committee: MS-Et/M.8P4.1/2014-2015

Keywords: Active management of third stage of labor, Uterine tonus assessment, Postpartum hemorrhage, Low and middle-income countries, Task shifting

\section{Background}

Postpartum hemorrhage is the leading cause of maternal death

Each year, approximately 287,000 women die because of preventable causes related to pregnancy and childbirth [1]. Almost all (99\%) of these deaths occur in low-income and middle-income countries (LMIC), with the largest burden in sub-Saharan Africa and South Asia [1].

The most common cause of maternal death is postpartum hemorrhage (PPH), defined as blood loss equal to or greater than $500 \mathrm{ml}$ within $24 \mathrm{~h}$ after birth of the infant. PPH occurs in around $10.5 \%$ of all deliveries and is responsible for more than 130,000 deaths annually [2, 3]. In sub-Saharan Africa, $33.9 \%$ of maternal deaths are due to $\mathrm{PPH}[4]$.

The maternal mortality ratio of Ghana in 2013 was 380 maternal deaths per 100,000 live births [5]. The leading cause of maternal deaths was obstetric hemorrhage, with estimates ranging between 27 and $32 \%$ [6-10].

Causes of PPH include uterine atony, uterine rupture, incisions, lacerations and coagulopathy, with uterine atony being the most common cause of PPH. Risk factors for atony include multiparity (five or more deliveries), polyhydramnios, fetal macrosomia, abnormal placentation, placental abruption, induced or prolonged labor and retained placental tissue $[2,11]$.

\section{AMTSL can prevent PPH}

Whether a woman dies from PPH depends largely on access to timely and competent obstetric care [12]. PPH mostly occurs during the third and fourth stages of labor $[2,13]$, and active management of the third stage of labor (AMTSL) can prevent its occurrence. AMTSL is recommended as a series of steps performed by skilled birth attendants (SBAs) by the World Health Organization (WHO) and the international professional organizations of gynecologists and midwives [2, 13, 14]. The steps include the provision of uterotonic drugs (oxytocin or misoprostol) immediately upon fetal delivery, controlled cord traction, massage of the fundus of the uterus immediately after placental delivery in the absence of uterotonics, and routine assessment of the uterine tonus every $15 \mathrm{~min}$ for the first $2 \mathrm{~h}$ postpartum.

\section{Task shifting in AMTSL}

AMTSL when performed by SBAs is the "gold standard" for prevention of $\mathrm{PPH}[2,15]$ and can reduce excessive blood loss by 50-70\% [16]. This provides a challenge in many LMIC where the majority of women live in rural areas and deliver at home without SBAs available, often assisted by traditional birth attendants or family members who are not educated in obstetric care [17]. Task shifting of AMTSL to community health workers, traditional birth attendants, or self-administration has been explored as a strategy to increase access to quality obstetric care in response to the obstacles [18].

Of all AMTSL interventions, uterotonic drugs are the most effective in preventing $\mathrm{PPH}$, although the relative contribution of each of the AMTSL components has not been well-studied [2, 19]. Oxytocin is the preferred uterotonic drug. However, as oxytocin is thermounstable, requires refrigeration and should be injected by SBAs, misoprostol is considered a safe, cheap and only slightly less effective alternative [20-23]. The WHO recommends the provision of misoprostol by community health workers for $\mathrm{PPH}$ prevention in rural areas and homebirths in absence of SBAs [2, 13]. The community distribution of misoprostol for self-administration by women is also widely explored as an option, but currently not recommended [24-26]. Controlled cord traction is contraindicated without assistance of a SBA [2]. To the best of our knowledge, there is currently no research or recommendation about the relative contribution of uterine tonus assessment for the prevention of $\mathrm{PPH}$ available, or the effect of task shifting of uterine tonus assessment $[2,13,27]$. Therefore, the aim of this non-inferiority randomized controlled trial (RCT) is to determine whether there is a difference in outcomes following routine uterine tone assessment every $15 \mathrm{~min}$ in the first $2 \mathrm{~h}$ postpartum performed by a SBA (midwife) compared to self-assessment by a patient.

\section{Methods/Design \\ Study design}

This study will be a pragmatic non-inferiority individual-level two-arm RCT comparing two strategies of AMTSL. 


\section{Participants}

The population base for the current study is pregnant women admitted in labor at Korle Bu Teaching Hospital (KBTH). KBTH has approximately 10,600 deliveries every year, approximately 200 per week. AMTSL has been implemented at KBTH. As a result of midwife understaffing, the routine assessment of the uterine tone for the first $2 \mathrm{~h}$ postpartum has been delegated to patients who received adequate training during the antenatal care (ANC) visits and who receive a repeated instruction after delivery. These women are regularly monitored by midwives. Yet, whether this task shifting is indeed equally effective has not been formally evaluated.

\section{Inclusion criteria}

In order to be eligible to participate in this study, the following criteria must be met: be at least 18 years of age, in labor with an expected vaginal delivery of a term singleton gestation, and recipient antenatal instructions on postnatal uterine tonus self-assessment.

\section{Exclusion criteria}

The following criteria will result in exclusion from study participation: operative delivery, severe anemia $(<8 \mathrm{~g} / \mathrm{dL})$ within 2 weeks of delivery, risk factors for PPH (history of previous $\mathrm{PPH}$, palpable uterine myoma, antepartum hemorrhage, anticipated breech delivery), intra uterine fetal death.

\section{Intervention}

Participants will be randomly assigned to one of the two arms:

1. uterine tonus assessment by patient, every $15 \mathrm{~min}$ for the first $2 \mathrm{~h}$ (current standard practice).

2. uterine tonus assessment by a SBA (midwife), every $15 \mathrm{~min}$ for the first $2 \mathrm{~h}$ (guideline recommended practice).

\section{Study objectives and hypothesis Primary objective}

To determine whether there is a difference in incidence of PPH ( $\geq 500 \mathrm{ml}$ blood loss) following routine uterine tone assessment every $15 \mathrm{~min}$ in the first $2 \mathrm{~h}$ postpartum performed by a SBA (midwife) compared to selfassessment by a patient.

\section{Secondary objective}

To determine whether routine uterine tonus assessment every $15 \mathrm{~min}$ in the first $2 \mathrm{~h}$ postpartum when performed by a SBA (midwife) compared to self-assessed by a patient, results in differences in incidence of severe PPH ( $\geq 1000 \mathrm{ml}$ blood loss), differences in mean blood loss, use of additional uterotonics, use of other procedures for management of PPH (surgery, manual placenta tissue removal), blood transfusion, maternal resuscitation and maternal death.

\section{Other study endpoints and parameters}

Neonatal outcomes will be assessed by: Apgar scores at 1 and 5 mins, neonatal resuscitation or perinatal death.

The following socio-demographic and obstetric characteristics will be collected: age, marital status, employment, education, number of previous pregnancies, number of previous deliveries (live births and still births), number of children, present pregnancy history, medical and surgical history.

\section{Hypothesis}

It is hypothesized that there will be no difference in outcomes for the SBA-assessed group compared to the selfassessment arm.

\section{Sample size calculation and justification}

$\mathrm{PPH}$ has an incidence of approximately $11.1 \%$ in the Ghanaian population [28]. The hypothesis of this noninferiority RCT is that there is no difference between the two treatments. To exclude a difference of between 5 to $7.5 \%$ with a 1 -sided alpha co-efficient of $5 \%$ at a power of $80 \%$, between 978 and 436 participants would have to be randomized [29]. Given resource restrictions, our sample size will be 800 women, and powered to exclude a difference of $5.53 \%$.

As the intervention and outcome assessment take place on the same day, we expect no loss to follow-up.

\section{Study procedures}

\section{Recruitment and enrollment}

Enrollment will take place at the ANC clinic and on the antenatal wards of KBTH after informed consent has been provided. Information about the study will be available at the ANC clinic and provided during the ANC education/training for uterine tonus assessment to familiarize women with the study (see Additional file 1 for the Standardized Operating Procedure for the training). Patients will be screened for eligibility based on the previously stated inclusion and exclusion criteria by trained research assistants. All patients will receive antenatal education on uterine tonus self-assessment.

\section{Randomization, treatment allocation, and treatment concealment}

Randomization occurs at presentation at the labor ward in established labor. If at this stage enrolled patients present with one of the exclusion criteria, they will be excluded from the study.

Participants will be assigned a study identification code based on consecutive enrollment. After the 
identification number has been assigned to a participant, a matching sealed opaque envelope in which the arm allocation is concealed can be opened by the research assistant. Women will be assigned an allocation arm while in labor, but before the onset of birth to facilitate the understanding of the arm they were allocated - as at time of full dilation or start of pushing, the ability to absorb the new information may have been reduced compared to an earlier stage of labor. The randomization list with blocks ranging between 2-6 identifiers will begenerated by the Data Management Department of the University Medical Center Utrecht using SAS software (SAS Inc., Cary, NC, USA). Additional randomization codes can be created to replace women who were excluded from the study because of complications, including caesarian sections.

Because of the nature of the intervention patients, care providers and research assistants will not be blinded.

\section{Blood loss estimation}

Assessment of blood loss will be performed by trained midwives by weighing blood collected after delivery of the placenta using disposable mats ("INCO pads") of 90 x $60 \mathrm{~cm}$. Scales will be standardized and calibrated. Blood loss will be measured up to $2 \mathrm{~h}$ postpartum.

\section{Other outcomes}

All other maternal endpoints will be obtained from maternal record books, surgery record books, antenatal booklet and interviews.

\section{Disposal of medical waste}

Mats used in the study will be disposed according to the standard procedures of disposal of medical waste at KBTH.

\section{Standard of care during study participation}

Participants will receive the standard of care during their participation in the study, including all standard care at $\mathrm{KBTH}$ associated with the development, prevention or treatment of PPH. Women who report inadequate uterine contraction to their midwife, will receive the standard of care according to PPH protocols of KBTH.

\section{Handling and storage of data and documents}

Data will be handled confidentially and anonymously through assigning a consecutive numeric identification (ID) code to participants. A separate subject identification list will be used to link the participant ID code to the participant names.

The local investigator(s) have access to the source data. The key to the code will remain with the coordinating investigator. In accordance to Good Clinical Practice rules, the dataset will be stored for 15 years.

\section{Statistical analysis}

Descriptive statistics for participant characteristics (age, marital status, employment, education, number of previous pregnancies, number of previous deliveries (live births and still births), number of children, present pregnancy history, medical and surgical history) will be presented as frequencies and percentages for dichotomous and categorical variables, and means and standard deviations for continuous variables will be presented. Differences between treatment groups will be assessed using Student's $t$ test for continuous outcome data and the chi square test for discrete outcome data, and reported significant with a $p$ value of $<0.05$.

Analyses for the first and second research objectives between intervention group and outcomes will be according to intention-to-treat. Primary and secondary dichotomous, categorical and continuous outcome variables will be descriptively analyzed in an approach similar to the participant characteristics analysis.

For the blood loss-related outcomes of interest (PPH, severe $\mathrm{PPH}$ and mean blood loss), linear and logistic regression analysis will be used to analyze the difference between the two arms. Participant characteristics with significant differences between the two groups despite randomization will be considered confounders in a multivariate logistic or linear regression analysis. The lower limit of the $90 \%$ confidence interval on the difference between the groups in frequency of the primary outcome will be used to assess non-inferiority for these three variables. Observations with missing outcome data will not be considered in these analyses.

\section{Ethical considerations}

The study will be conducted according to the principles of the Declaration of Helsinki and in accordance with the Dutch Law Medical Research Involving Human Subjects Act and Good Clinical Practice. The trial has been approved by the University of Ghana Medical School Ethical and Protocol Review Committee (MS-Et/M.8P4.1/2014-2015). After information is provided in Twi or English, participants need to provide written informed consent (signature or thumb print) before enrollment.

To ensure participant's rights are protected, an independent gynecologist will be available for counseling.

\section{Benefits and risks assessment}

There are no direct benefits or incentives for participants who participate in this study. As the intervention group receives a midwife assessment of uterine tonus, the benefit of participating in this study is that PPH may be detected slightly earlier than may otherwise be the case. As the study takes places at the labor ward, the control arm will receive the same quality of care and will be monitored by the midwives as part of routine care. 
Because most of the data will be collected from collection books, the time investment is minimized as much as possible.

All participating women are enrolled in the national health insurance scheme. No data safety monitoring board was installed for this study. Details about safety reporting are available upon request.

\section{Public disclosure and publication policy}

This trial has been publicly registered with the PanAfrican Clinical Trials Registry (PACTR, PACTR201402000736158) and Clinicaltrials.gov (NCT02223806). Scientific publications will be developed under the direction of the investigators and disclosed unreservedly in international scientific peer-reviewed journals. The full protocol is available upon request.

\section{Discussion}

\section{Diagnosis of PPH}

Despite the diagnosis of PPH being dependent on the measurement of blood loss the professional organizations of gynecologists/obstetricians and midwives [14] and WHO [2] do not have a recommended standardized method to measure blood loss. Because visual estimation has been established to be inaccurate, especially with larger amounts of blood loss, other more objective methods to diagnose PPH have been developed. These methods are direct measurement, gravimetry and photometry. With direct measurement, blood is directly collected in a pan or collector bag. With photometry, the amount of blood loss can be determined by a process to convert blood pigment to alkaline hematine and subsequently analyzed. This method is referred to as the "gold standard." However, in low resource settings the availability of this photometric method will be low and the time needed to extract all blood from linen and pads will take too long to diagnose PPH. A method commonly used in clinical practice as an alternative is gravimetry in which standard-sized pads (e.g. "INCOpads," 90 x $60 \mathrm{~cm}$ ) soaked with blood are weighed after delivery of the placenta, and grams converted to milliliters of blood loss. Although this method is accurate in diagnosing $\mathrm{PPH}$, it can overestimate the incidence of $\mathrm{PPH}$, because it is difficult to discriminate blood from other fluids like urine or amniotic fluid [30]. In this study, the gravimetric method for diagnosing $\mathrm{PPH}$ will be applied.

We acknowledge that this study's sample size and (clinical) setting may not allow for immediate generalizability to other settings. Instead, larger studies which include other clinical settings and non-clinical settings will be necessary to externally validate the findings before implementation. Future research could also include the relative contribution of tonus assessment to the prevention of $\mathrm{PPH}$, the optimal training program and timing of training.
The experiences of pregnant women and health providers in task shifting of tonus assessment could also be considered in future studies.

\section{Trial status}

The study will take place from April 2014 until July 2015 at the labor ward of Korle Bu Teaching Hospital in Accra, Ghana. Enrollment commenced in April 2014.

\section{Additional file}

Additional file 1: Script of antenatal clinic health talk. (DOC $23 \mathrm{~kb}$ )

\section{Abbreviations}

AMTSL: active management of the third stage of labor; ANC: antenatal care; CCMO: Dutch Central Committee on Research Involving Human Subjects; ID: identification; KBTH: Korle Bu Teaching Hospital; LMIC: low-income and middle-income countries; PACTR: Pan-African Clinical Trials Registry; PPH: postpartum hemorrhage; RCT: randomized controlled trial; SBA: skilled birth attendant; UTAMP: Uterine Tonus Assessment by Midwives versus Patient; WHO: World Health Organization.

\section{Competing interests}

The authors declare that they have no competing interests.

\section{Authors' contributions}

JLB conceived of the study, participated in its design and coordination of the study, and drafted the first version of the manuscript. NKRD conceived the research question, participated in its design and coordination of the study, and participated in the drafting of the manuscript. KKG, EvdL, DEG, AF, and MR participated in the design of the study and helped to draft the manuscript. RA participated in the design of the study. TR, ETM, RD contributed to the design and coordination of the study and helped to draft the manuscript. All authors have approved the final version of the manuscript.

\section{Authors' information}

Principal investigators: JB trained as a medical doctor and epidemiologist. NKRD is a gynecologist and senior lecturer at the Medical School of the University of Ghana. KKG is an epidemiologist and associate professor of global health at the UMC Utrecht.

\section{Acknowledgments}

We would like to thank the Department of Woman and Baby of the University Medical Center Utrecht and Share-Net International for their financial contribution to realize this study. The Julius Center for Health Sciences and Primary Care at the University Medical Center Utrecht and the Korle Bu Teaching Hospital contributed financially to this study by making the authors available to contribute to this study.

We would like to express our appreciation to the Dutch Central Committee on Research Involving Human Subjects (CCMO) for providing a protocol template. Prof. René Eijkemans is recognized for his advice for the statistical analyses.

\section{Author details}

${ }^{1} J$ ulius Global Health, Julius Center for Health Sciences and Primary Care, University Medical Center Utrecht, Heidelberglaan 100, 3584CX, Utrecht, The Netherlands. ${ }^{2}$ School of Medicine and Dentistry, College of Health Sciences, University of Ghana, Accra, Ghana. ${ }^{3}$ Department of Obstetrics and Gynecology, Korle Bu Teaching Hospital, Accra, Ghana. ${ }^{4}$ School of Public Health, College of Health Sciences, University of Ghana, Accra, Ghana. ${ }^{5} \mathrm{WHO}$ Country Office Ghana, World Health Organization, Accra, Ghana.

${ }^{6}$ Department of Obstetrics and Gynecology, University Medical Center Utrecht, Utrecht, The Netherlands. 'Division of Epidemiology and Biostatistics, School of Public Health, Faculty of Health Sciences, University of the Witwatersrand, Johannesburg, South Africa. 
Received: 8 May 2015 Accepted: 8 December 2015

Published online: 18 December 2015

\section{References}

1. Say L, Chou D, Gemmill A, Tunçalp Ö, Moller AB, Daniels J, et al. Global causes of maternal death: a WHO systematic analysis. Lancet Glob Heal. 2014:2:e323-33.

2. World Health Organization. WHO recommendations for the prevention and treatment of postpartum haemorrhage. Geneva: World Health Organization Press; 2012.

3. McLintock C, James AH. Obstetric hemorrhage. J Thromb Haemost. 2011;9: 1441-51.

4. Khan KS, Wojdyla D, Say L, Gülmezoglu AM, Van Look PF. WHO analysis of causes of maternal death: a systematic review. Lancet. 2006;367:1066-74.

5. WHO, UNICEF, UNFPA the World Bank and the United Nations Population Division. Trends in maternal mortality: 1990 to 2013. Geneva; 2014

6. Zakariah AY, Alexander S, van Roosmalen J, Buekens P, Kwawukume EY, Frimpong P. Reproductive age mortality survey (RAMOS) in Accra, Ghana. Reprod Health. 2009;6:7.

7. Ghana Health Services. Annual Reproductive and Child Health, Report 2008. 2008

8. Ghana Health Services. Annual Reproductive and Child Health, Report 2009. 2009

9. Ghana Health Services. Annual Reproductive and Child Health, Report 2011. 2011.

10. Ghana Health Services. Annual Reproductive and Child Health, Report 2012. 2012.

11. Stanton C. Use of active management of the third stage of labour in seven developing countries. Bull World Health Organ. 2009;87:207-15.

12. Ronsmans C, Graham WJ. Maternal mortality: who, when, where, and why. Lancet. 2006:368:1189-200.

13. WHO. WHO recommendations on prevention and treatment of postpartum haemorrhage - highlights and key messages from new 2012 global recommendations. Geneva: World Health Organization; 2014.

14. International Federation of Gynecology and Obstetrics, The International Confederation of Midwives. Joint statement: management of the third stage of labour to prevent post-partum haemorrhage. J Midwifery Womens Health. 2004:49:76-7.

15. Sheldon WR, Blum J, Vogel JP, Souza JP, Gülmezoglu AM, Winikoff B. Postpartum haemorrhage management, risks, and maternal outcomes: findings from the World Health Organization Multicountry Survey on Maternal and Newborn Health. BJOG. 2014;121(Suppl):5-13.

16. Begley C, Gyte G, Devane D, McGuire W, Weeks A. Active versus expectant management for women in the third stage of labour. Cochrane Database Syst Rev. 2011. doi:10.1002/14651858.CD007412.pub3.

17. Prata N, Bell S, Holston M, Quaiyum MA. Is attendant at delivery associated with the use of interventions to prevent postpartum hemorrhage at home births? The case of Bangladesh. BMC Pregnancy Childbirth. 2014:14:24.

18. World Health Organization. WHO recommendations: optimizing health worker roles to improve access to key maternal and newborn health interventions through task shifting. Geneva; 2012.

19. Prata N, Bell S, Weidert K. Prevention of postpartum hemorrhage in lowresource settings: current perspectives. Int J Womens Health. 2013:5:737-52.

20. Miller $S$, Lester $F$, Hensleigh P. Prevention and treatment of postpartum hemorrhage: new advances for low-resource settings. J Midwifery Womens Health. 2004;49:283-92.

21. Mobeen N, Durocher J, Zuberi N, Jahan N, Blum J, Wasim S, et al. Administration of misoprostol by trained traditional birth attendants to prevent postpartum haemorrhage in homebirths in Pakistan: a randomised placebo-controlled trial. BJOG. 2011;118:353-61.

22. Tunçalp Ö, Hofmeyr GJ, Gülmezoglu AM. Prostaglandins for preventing postpartum haemorrhage. Cochrane database Syst Rev. 2012;8:CD000494.

23. Gülmezoglu AM, Villar J, Ngoc NT, Piaggio G, Carroli G, Adetoro L, et al. WHO multicentre randomised trial of misoprostol in the management of the third stage of labour. Lancet. 2001;358:689-95.

24. Oladapo OT, Fawole B, Blum J, Abalos E. Advance misoprostol distribution for preventing and treating postpartum haemorrhage. Cochrane database Syst Rev. 2012;2:CD009336

25. Prata N, Passano P, Bell S, Rowen T, Potts M. New hope: community-based misoprostol use to prevent postpartum haemorrhage. Health Policy Plan. 2013;28:339-46.
26. Geller S, Carnahan L, Akosah E, Asare G, Agyemang R, Dickson R, et al Community-based distribution of misoprostol to prevent postpartum haemorrhage at home births: results from operations research in rural Ghana. BJOG. 2014;121(3):319-25. http://www.ncbi.nlm.nih.gov/pubmed/ ?term=Communitybased+distribution+of+misoprostol+to+prevent +postpartum+haemorrhage+at+home+births\%3A+results+from+operations +research+in+rural+Ghana

27. Weeks A. The prevention and treatment of postpartum haemorrhage: what do we know, and where do we go to next? BJOG. 2015;122(2):202-10.

28. Stanton CK, Newton S, Mullany LC, Cofie P, Tawiah Agyemang C, Adiibokah E, et al. Effect on postpartum hemorrhage of prophylactic oxytocin (10 IU) by injection by community health officers in Ghana: a community-based, clusterrandomized trial. PLoS Med. 2013;10:e1001524.

29. Sealed Envelope Ltd. 2012. Power calculator for binary outcome noninferiority trial. [Online] Available from: https://www.sealedenvelope.com/ power/binary-noninferior/. Accessed 21 September 2015.

30. Schorn MN. Measurement of blood loss: review of the literature. J Midwifery Womens Health. 2010:55:20-7.

\section{Submit your next manuscript to BioMed Central and we will help you at every step:}

- We accept pre-submission inquiries

- Our selector tool helps you to find the most relevant journal

- We provide round the clock customer support

- Convenient online submission

- Thorough peer review

- Inclusion in PubMed and all major indexing services

- Maximum visibility for your research

Submit your manuscript at www.biomedcentral.com/submit 